\title{
The dynamic changes in cytokine responses in COVID-19: a snapshot of the current state of knowledge
}

\author{
"The role of cytokines in COVID-19" online symposium was presented on 18 June 2020 by the NIH/FDA \\ Immunology and Cytokine Interest Groups and was purposed to discuss our rapidly changing understanding \\ of COVID-19-related cytokine responses in different stages of infection, including the etiologies, downstream \\ consequences and possible mitigation strategies. The recording is available at https://nci.rev.vbrick.com/ \\ sharevideo/03106730-66cc-47ba-870b-f6e6274a998a.
}

$\mathrm{T}$ he symposium was opened by Anthony Fauci, Director of the National Institute of Allergy and Infectious Diseases at the US National Institutes of Health (NIAID, NIH), and Janet Woodcock, Director of the Center of Drug Evaluation and Research, Food and Drug Administration (CDER, FDA) and currently leading the therapeutics component of Operation Warp Speed. Fauci briefly reviewed the current status of the coronavirus disease 2019 (COVID-19) pandemic, noting that the worldwide incidence had grown to 8 million cases and more than 300,000 deaths, with $>120,000$ fatalities in the USA alone (incidence on June 18 2020). The causative virus, the severe acute respiratory syndrome coronavirus 2 (SARS-CoV-2), is a single-stranded RNA virus that uses angiotensin-converting enzyme 2 (ACE2) as a cellular receptor. The atomic-level conformation of the prefusion spike protein of the virus was recently described by NIAID Vaccine Research Center scientists and colleagues ${ }^{1}$. He also underscored the role of cytokines in the pathogenesis of the different clinical presentations of COVID-19, ranging from asymptomatic to pneumonia, neurological disorders, acute respiratory distress syndrome (ARDS), cardiomyopathies, sepsis, hypercoagulability, multiorgan failure and death, as well as the multisystem inflammatory syndrome seen in children. Also, the benefit of dexamethasone treatment in severe COVID19 cases requiring ventilation was discussed, which is consistent with the central roles of inflammation and a cytokine storm in causing serious pathology. Fauci ended his talk by calling attention to the multiple initiatives undertaken and supported by the NIAID to address the COVID-19 outbreak. Woodcock followed with an address that underscored the broad variety of clinical presentations of COVID-19, thus highlighting the central role of the immune response in this disease. She also remarked on the apparent geographic clusters of disease manifestations and the need to better understand possible factors in host-pathogen interactions beyond those health conditions already identified, such as prior innate immune experience and subtle differences in ACE2 expression in the populations. Lastly, she discussed the complexity of the data emerging from the multiple clinical trials that are targeting the inflammatory process underlying the disease, emphasizing the importance of establishing clinically relevant biomarkers to guide the therapeutic course.

\section{Cytokine response and disease severity}

The first scientific session of the meeting focused on the changing cytokine response over the course of COVID-19 and was opened by Miriam Merad (Mount Sinai School of Medicine). Merad first reminded us that the infection is asymptomatic in $80 \%$ of adults and most children, but $20 \%$ of patients require hospitalization in the intensive care unit (ICU). The mortality rate for patients in the ICU is $25 \%$, with most deaths attributed to severe inflammation and embolic complications. In agreement with Woodcock's remarks on the importance of biomarkers to better target therapeutic efforts, Merad described studies using high-dimensional profiling to identify early markers that predict disease severity. In one study, her group selected a platform that monitors interleukin (IL)6 , IL-8, IL-1 $\beta$ and tumor necrosis factor (TNF), which are well-established targets for anti-inflammatory therapeutics. They tested over 1,500 patients on the day of hospitalization and then correlated the serum cytokine concentrations with disease outcome. Her data showed that IL-6, IL-8 and TNF, and to a lesser extent, IL- $1 \beta$, were elevated at the time of hospitalization, and their concentrations correlated with disease outcome and mortality, even after correcting for age, ethnicity, race and comorbidities, suggesting that they could be used to identify patients at risk of severe disease ${ }^{2}$.

The proinflammatory cytokines remained elevated throughout the disease course unless patients were treated with steroids or remdesivir, which reduced the level of circulating IL-6. She also stressed that IL- 6 and TNF are regulated independently and thus could be targeted in parallel in patients with severe disease. Merad then described early results from deep-profiling longitudinal studies using whole-blood proteomics that identified 23 clusters of cytokines that are differentially expressed in patients with mild disease, severe disease without end organ damage and severe disease with end organ damage. In this broader assessment, patients with severe disease had increased IL- 6 and other proinflammatory cytokines, while those with moderate disease had a pattern that suggested T cell priming. Lastly, Merad suggested that the pattern of cytokine response in children with multisystem inflammatory syndrome partly resembled that of adults with severe disease, as they had increased expression of proinflammatory cytokines; however, they also had a variety of autoantibodies, including some classical autoantibodies, but also autoantibodies directed to cardiac and endothelial antigens that might explain the disease manifestations. Importantly, the cytokine patterns that were present at the time of hospitalization in children as well as adults were maintained throughout the disease course unless modified by treatment with 
steroids, remdesivir or immunomodulatory therapy and could be correlated with disease outcome, suggesting that they can be used to guide the therapeutic approach.

John Tsang (NIAID, NIH) then spoke of the application of a systems immunology analysis of COVID-19. He described studies employing cellular indexing of transcriptomes and epitopes by sequencing (CITE-seq), a single-cell analysis method that combines highly multiplexed surface protein marker detection with transcriptome profiling. The Tsang lab and collaborators evaluated peripheral blood mononuclear cells (PBMCs) from a longitudinal cohort of hospitalized patients with COVID-19 from Brescia, Italy, taken at the height of the outbreak in that region. The patients evaluated in this first experiment included 13 severe or critical patients and 5 ageand sex-matched healthy controls. Two observations in particular emerged that differed from findings reported by other investigators. First, whereas previous studies suggested that the virus disrupted the type I interferon (IFN) signaling pathway, fueling speculation that the lack of an early type I (and III) IFN response permits rapid viral spread and induces hyperinflammatory responses, this study identified a clear type I IFN signature across major immune cell subsets in patients with COVID-19. These findings suggest that a careful evaluation of the patient populations scrutinized across different studies should be performed, as the timelines for the induction of such responses may differ according to disease stage and may be further influenced by genetic and environmental factors. Furthermore, thus far, Tsang has found no evidence of $\mathrm{T}$ cell exhaustion, as the expanded $\mathrm{CD}^{+} \mathrm{T}$ cell clones were found to have decreased PD-1 expression in comparison with the non-expanded cells in patients. This observation again differs from other studies that found high amounts of PD-1 expression on lymphocytes ${ }^{3}$, indicating exhaustion and raising the possibility that checkpoint blockade should be considered for treatment.

The final seminar in this session was given by Frank van de Veerdonk (Radboud University Medical Center). The main focus of his seminar regarded unique aspects of COVID-19 immune pathology; specifically, the role of ACE2 in modulation of the kallikrein-kinin system. Preliminary results suggested that this system has a role in the angioedema observed in the lungs of COVID-19 patients. Biological pathways were evaluated via proteomic analysis of serum from patients with severe (necessitating ICU care) versus more moderate (not requiring ICU care)

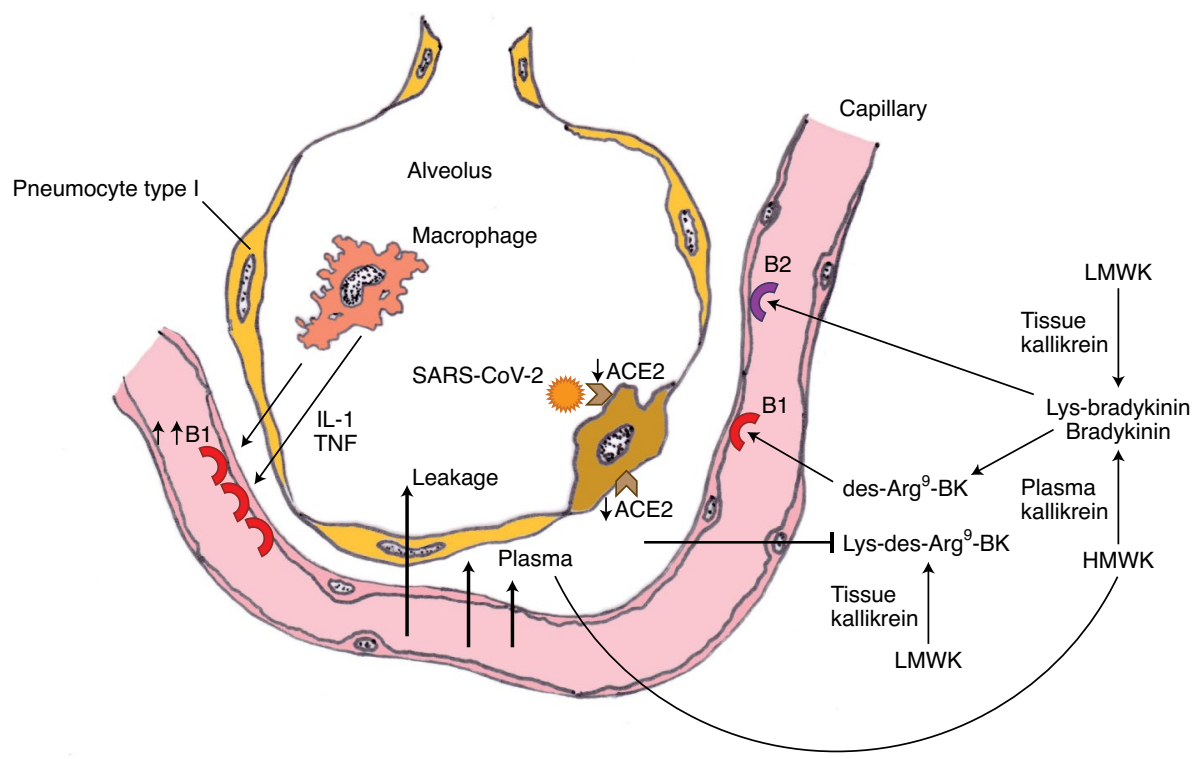

Fig. 1 | Alveolus during severe COVID-19 hyperinflammation. ACE2 downregulation by SARS-CoV-2 is followed by the loss of neutralizing capacity of Lys-[des-Arg ${ }^{9}$ ]-bradykinin (BK) in the lung, leading to plasma leakage. Subsequently, plasma leakage results in more B1R ligands (des-Arg9-BK) and B2R ligands (bradykinin), enhancing vascular permeability and angioedema. CPM, carboxypeptidase $M$; CPN, carboxypeptidase N; HMWK, high-molecular-weight kininogen; LMWK, low-molecular-weight kininogen. Figure reproduced with permission from ref. ${ }^{4}$, eLife Sciences Publications.

disease, with an initial focus on IL-1 biology and autoinflammation and a subsequent focus on the kallikrein-kinin system. IL-6 upregulation was prominent in patients with severe disease, which van de Veerdonk proposed was a manifestation of the strong induction of an autoinflammatory loop via IL- $1 \beta$ and the IL-1 receptor (IL$1 \mathrm{R})$. He proposed that, following SARS $\mathrm{CoV}-2$ binding to pathogen recognition receptors, pro-IL- $1 \beta$ is induced, processed to IL- $1 \beta$ by activated inflammasomes and stimulates IL- 6 and IL- 18 production (from pro-IL-18). IL-1 $\beta$ binds to IL-1R on monocytes/macrophages, generating the autoinflammatory loop and recruitment of polymorphonuclear leukocytes. He also noted that IL- $1 \alpha$ released during tissue damage may further contribute to the autoinflammatory loop by binding and signaling through IL-1R, inducing more IL- $1 \beta$. He further remarked that IL- 1 is a difficult cytokine to measure (echoing others) and that concentrations in plasma likely do not reflect those in the lung, suggesting that bronchoalveolar lavage (BAL) measurements would be important in verifying its upregulation at the site of primary pathology. Lastly, he remarked on the substantial reductions in IL-7R and stem cell factor and their potential relevance to the commonly observed lymphopenia in patients with severe disease, which is of clear interest from a therapeutic standpoint. In the last part of his talk, van de Veerdonk focused on the reduced amounts of serpin family A member 12 (SERPINA12) and DPP4 (CD26) in patients in the ICU versus those not in the ICU. Since these factors suppress inflammation mediated by kallikreins and it is expected that there is positive, coordinated stimulation of the kallikreinkinin system from increases in IL-1 and IL-6 as well as from activated complement, the upregulation of the kallikrein-kinin system was investigated. Most critically, kininogens processed by kallikrein, a serine protease, will generate bradykinin (BK) or Lys-BK (Fig. 1), which can be further metabolized by tissue carboxypeptidases, producing des-Arg'-BK (DABK). DABK binds to bradykinin 1 receptors (B1Rs) and enhances vascular permeability, which can lead to local angioedema. Critically, DABK is inactivated by ACE2. Because ACE2 is internalized by SARS-CoV-2, it is no longer present to counter the downstream effects of DABK, thus producing angioedema in the lung, which potentially explains the early ARDS manifestation in these patients. Furthermore, IL-1 and inflammation in general stimulate increased expression of B1Rs, thus upregulating these receptors in the inflamed lung and boosting tissue-level 


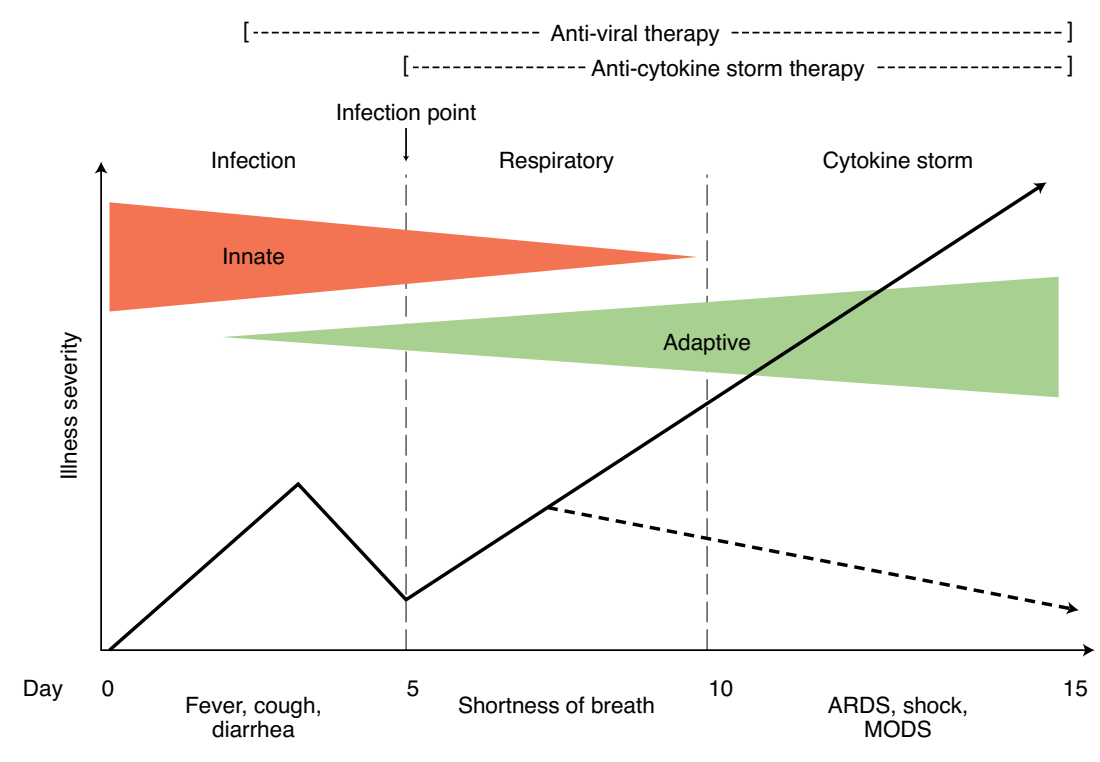

Fig. 2 | The COVID-19 inflection point of illness. A graphic depiction of the course of illness for the up-to-20\% of individuals with COVID-19 who develop cytokine storm syndrome (CSS) and respiratory distress requiring hospitalization ${ }^{17}$. Severity of illness is presented along the $y$ axis and time in days along the $x$ axis. The participation of both the innate and adaptive immune responses is presented as orange and green triangles, respectively, during the different phases of disease. The early stages of infection ( $<5$ days of symptoms) give rise to a more prominent respiratory phase in those with early signs of CSS, such that an inflection point of illness occurs typically between days 5 and 7 of illness. This interval is the time in which targeted immunomodulatory therapy will probably be most beneficial in lowering mortality (dashed arrow). MODS, multiorgan dysfunction syndrome. Figure adapted with permission from ref. ${ }^{17}$, the Journal of Rheumatology Publishing.

angioedema. The ensuing plasma leakage into the alveolar space allows activation of plasma kallikrein-kinin at the site of infection. Subsequent production of bradykinin results in persistent angioedema via bradykinin activation of the bradykinin 2 receptor (B2R).

This observation led to the hypothesis that the kallikrein-kinin system was critically involved in lung pathology in COVID-19, which was tested by treating patients with hypoxia and enhanced oxygen requirements with icatibant, an approved B2R blocker for hereditary angioedema (HAE). Promising effects were observed, which will be published soon. This finding suggests the need for expanded trials as well as the investigation of a longer lasting agent, lanadelumab, which blocks plasma kallikrein activity and is approved for HAE. In light of the findings of this study and data from other sources, a treatment protocol based on disease stage was proposed ${ }^{4}$.

\section{Cellular origins of cytokines}

The second session commenced with Chen Dong (Tsinghua University), who discussed his recently published work ${ }^{5}$ on humoral responses in patients with COVID-19. In a small cohort, his group readily detected
IgG1 and IgM antibodies that recognized recombinant SARS-CoV-2 nucleoprotein and the receptor-binding domain of the spike protein (S-RBD) in patient sera as compared to serum from healthy individuals. In general, antigen-specific antibody concentrations were higher in individuals recently released from hospital (8 patients) as compared to those assayed 2 weeks after release (6 patients). Longitudinal studies on larger patient cohorts will be needed to determine the time course and duration of humoral responses during COVID-19. Somewhat consistent with these observations, Luigi Notarangelo (NIAID, NIH) noted in a study of more than 300 patients from Brescia and Monza in northern Italy that the proportion of plasmablasts in blood was highest in the early stages of COVID-19 and decreased with disease course ${ }^{6}$. Using a pseudovirus particle-based neutralization assay, Dong found that 13 out of 14 patients developed varying levels of neutralizing antibodies with specificity for S-RBD. In an independent study, $94 \%$ of 175 patients developed neutralizing antibodies, suggesting that such humoral responses are a consistent feature of COVID-197. Importantly, antibody titers did not correlate with disease duration in this study, leaving open the question of the contribution of antibodies in determining disease progression. It is possible that a threshold level of antibody synergizes with other features of immune (for example, $\mathrm{T}$ cell activation and memory formation) and inflammatory responses to affect disease resolution. It also remains unclear to what extent neutralizing antibodies persist in convalescent individuals and whether they will protect upon rechallenge with SARS-CoV-2. In this regard, another study reported that $40 \%$ of asymptomatic individuals and $13 \%$ of symptomatic individuals became seronegative 8 weeks after discharge from hospital ${ }^{6}$. This apparently short-lived antibody response contrasts sharply with detectable SARS-CoV-specific IgG 2 years after infection ${ }^{8}$.

Notarangelo provided a comprehensive overview of cellular and cytokine changes in the context of disease course and severity in the Italian cohort. A recurring pattern in COVID-19 is the increased expression of proinflammatory cytokines such as IL-6, TNF and IL- $1 \beta$, as well as an IFN- $\gamma$ signature evidenced by expression of the downstream cytokine CXCL9; similar observations were also presented by Merad and Tsang in Session 1. Serum concentrations of soluble biomarkers of endothelial cell activation (ICAM-1 and VCAM-1) and of septic shock (lipopolysaccharide binding protein (LBP) and sIL-33R) were also significantly elevated in patients with COVID-19 and more so in those with critical disease who eventually died. In an interesting twist, Notarangelo showed that, during the course of disease, certain inflammatory markers, such as IL-6, did not change significantly, whereas others, such as soluble IL-33R (sIL-33R) and CXCL10, decreased in patients who eventually recovered but remained persistently elevated in those who succumbed to COVID19. Additionally, cytokines associated with myeloid differentiation positively correlated with disease severity, suggesting a contribution of de novo myelopoiesis. A striking observation in this study was the reduced numbers of $\mathrm{CD} 4^{+}$and $\mathrm{CD}^{+}$ $\mathrm{T}$ cells in patients with COVID-19. T lymphopenia was evident even in patients with mild symptoms, and it reached extreme deficits in those with severe disease ${ }^{9}$. The underlying cause of $\mathrm{T}$ cell loss is not known and will be an important area of future research. Notarangelo also pointed out the surprising decrease in soluble Fas ligand (sFasL) and sCD62L, contrasting with high concentrations of sCD25, a biomarker of $\mathrm{T}$ cell activation. A preprint in bioRxiv also reported profound antigen-specific $\mathrm{T}$ cell 

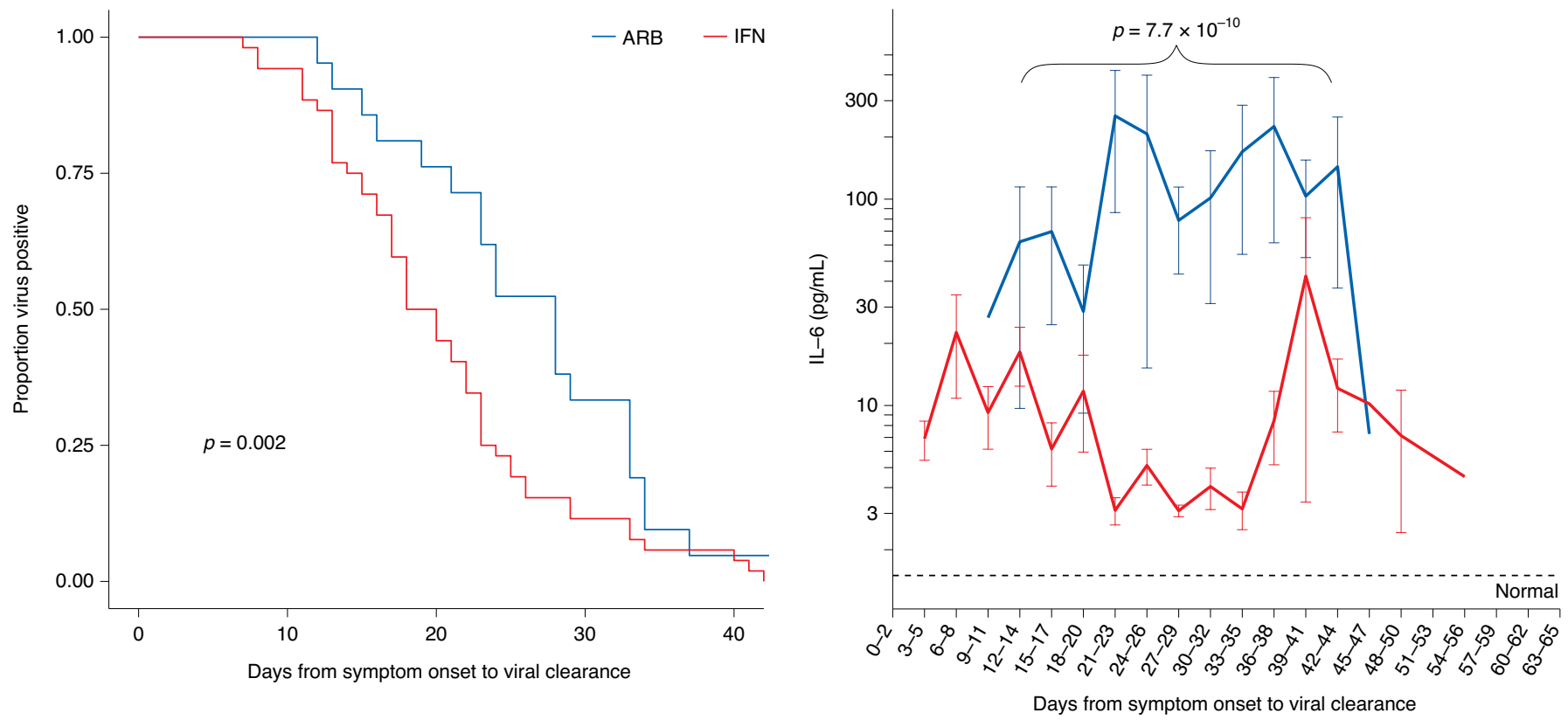

Fig. 3 | IFN- $\mathbf{\alpha} \mathbf{2 b}$ treatment accelerates viral clearance and reduces inflammatory IL-6. Patients with confirmed COVID-19 were treated with either ARB alone (ARB; 24 patients) or IFN- $\alpha 2 b$ with or without ARB (IFN; 53 patients). Left, upper respiratory samples were assessed by PCR for the presence of SARS-CoV-2. Shown is the proportion of patients who had detectable virus as a function of the day of sampling from symptom onset. The $P$ value for treatment effect was assessed using a Cox proportional-hazards model that included age and comorbidities as covariates. Right, patients were serially sampled for assessment of IL- 6 from the day of symptom onset. Values recorded were aggregated across 3 day intervals and shown as the mean \pm s.e. $P$ value was assessed using $R$ v.3.6.0, and analysis of variance (ANOVA) was used to test for treatment effect, adjusting for age and comorbidities. Figure reproduced with permission from ref. ${ }^{15}$, International Union of Immunological Societies.

activation and cytotoxic responses even in seronegative individuals ${ }^{7}$. Further studies are required to understand the contributions of such contrasting immune responses to the course of COVID-19. Finally, Notarangelo provided evidence that reduced expression of HLA-DR and CD4 on peripheral blood monocytes (the former a biomarker of antigen-presenting capacity) correlated with disease severity ${ }^{9}$. By impairing antigen presentation and the consequent activation of T cells, this cellular phenotype could attenuate effective responses during SARS-CoV-2 infection. The predictive potential of this biomarker remains to be determined.

The final speaker in this session, Xiaoyu $\mathrm{Hu}$ (Tsinghua University), elaborated specifically on the nature and origins of cytokine responses in COVID-19, focusing on the lung myeloid compartment of patients. Previous studies have shown that the largest population of lung-resident alveolar macrophages are of fetal origin and are maintained by proliferation in $s \mathrm{u}^{10}$. Single-cell RNA sequencing studies of BAL fluid from patients with COVID-19 showed a substantial influx of peripheral monocyte-derived macrophages to the lungs, with a proportionate reduction in the frequency of alveolar macrophages; these changes in cell populations correlated with disease severity ${ }^{11,12}$. Recruited macrophages primarily expressed high amounts of chemokines such as CCL2, CCL7 and CCL8 and, with increasing disease severity, CXCL10 and CCL3. By contrast, typical proinflammatory cytokines such as IL- 6 and IL-8 that were elevated in the periphery were not markedly upregulated in these cells. The distinct lung-specific responses may contribute to COVID-19 lung pathophysiology, for example, by CCL2-mediated recruitment of macrophages $^{11}$.

The basis for the dichotomy between the chemokine-dominated lung macrophage response and the peripheral prevalence of more classical inflammatory cytokines is not clear. One possibility is that the lung microenvironment skews macrophage responses toward chemokine gene expression. In this scenario, lung macrophage responses in other acute respiratory syndromes should also be similarly skewed. However, it should be noted that resident alveolar macrophages do not exhibit this pattern, indicating that it is not solely a microenvironment issue. Alternatively, SARS-CoV-2 infection may generate a unique inflammatory milieu that promotes the observed gene expression pattern. That it is restricted to recruited macrophages hints at a role for peripheral priming in generating the phenotype.

\section{Cytokine-targeted mitigation strategies}

The symposium concluded with a session that discussed the recent advances in managing both the virus infection and the associated cytokine storm using biologics and drugs to treat the disease and how such approaches significantly ameliorated disease outcomes. Randy Cron (University of Alabama at Birmingham) started off this session by reminding us that, long before COVID-19 came into the limelight, the cytokine storm was already recognized as a major issue in the field of immune homeostasis. Cytokine storm is an umbrella term for several hyperinflammatory immune responses that include cytokine release syndrome, culture-negative sepsis, macrophage activation syndrome and hemophagocytic lymphohistiocytosis (HLH), among others. Therefore, there are several causes of cytokine storm, which can be triggered by genetic factors, cancer, viral infections and other insults and that need to be managed accordingly. Consequently, the COVID-19-triggered cytokine storm should be manageable using knowledge amassed in the past based on the management of similar viral infections (Fig. 2). In the course 
of his brief but cutting-edge overview of the literature, Cron cited a previous study wherein the inability of perforin-deficient CD8 ${ }^{+}$CTLs to eliminate LCMV-infected target cells resulted in the death of infected mice by hyperactivation, but not when IFN- $\gamma$ was neutralized ${ }^{13}$.

Along these lines, the disruption of other genes involved in the cytolytic pathway also results in a cytokine storm and lethal hyperinflammation. For example, the Cron group performed whole-exome sequencing of patients who succumbed to H1N1 influenza infection and found that $36 \%$ of the 14 individuals in the study had mutations in the genes encoding either perforin or the lysosomal trafficking regulator (LYST). Thus, cytokine storm is evidently associated with the failure to clear virus-infected target cells and persistent $\mathrm{T}$ cell stimulation. As a solution, Cron summarized a few approaches focused on targeting IL-6, IL-1 or JAK/STAT proteins to suppress the cytokine storm. He concluded his talk by offering alternative strategies to reduce hyperinflammation, such as JAK inhibitors and glucocorticoids. Specifically, the World Health Organization initially recommended against the use of corticosteroids for COVID-19 based on the experiences of SARS and MERS, but recent data showed a clear reduction in the need for ventilation and in mortality in patients with COVID-19 treated with dexamethasone ${ }^{14}$. Steroids are widely available and affordable, and, with careful dosing and timing, these agents could become powerful tools in fighting the cytokine storm triggered by SARS-CoV-2.

In the next seminar, Eleanor Fish (University of Toronto) put the COVID19 pandemic into greater perspective by pointing out two other coronavirus outbreaks that had preceded SARS-CoV-2, namely SARS-CoV in 2002 and MERS-CoV in 2012. To control viral infections, the first 24-72 hours are the most critical, and Fish emphasized that the type I IFN response by innate cells plays a pivotal role in this process. Type I IFNs are powerful because they not only act directly on infected cells to suppress viral replication but also recruit and activate immune cells to clear the virus. Notably, the SARS-CoV-2 genome encodes genes that suppresses IFN production, such as Nsp1, Nsp3, ORF6 and the M proteins.

Thus, it is evident that viruses are equipped with tools to dampen host IFN production, and both SARS-CoV-2 and SARS-CoV fail to trigger a good type I IFN response.

Along these lines, the Fish group demonstrated that SARS-CoV replication in vitro could be effectively suppressed by IFN alfacon-1 treatment (a synthetic
IFN- $\alpha$ ) and that such an effect was further reproduced in a clinical trial when SARS patients were subcutaneously injected with IFN alfacon-1, resulting in rapid lung clearance and substantial improvements in various clinical parameters. Translating those findings to COVID-19, a clinical trial was designed involving 77 hospitalized patients with confirmed cases of COVID19 in Wuhan, China. In this trial, patients were treated with either the antiviral drug arbidol (ARB), nebulized IFN- $\alpha 2 b$ or a combination of the two agents. While the investigators did not observe any differences among patients in the treatment groups with respect to body temperature, oxygen saturation or blood biochemistry, there was a significant difference in viral clearance, as IFN-treated patients cleared the virus much faster. Notably, IFN-treated patients also showed significantly reduced concentrations of serum IL-6 and C-reactive protein (CRP) (Fig. 3$)^{15}$. Thus, with the caveat that this trial was a non-randomized, small-cohort study, the data advocated for using type I IFN as an early intervention agent in COVID-19. Moreover, type I IFN treatment may prove to be more beneficial than other treatments because it did not trigger a cytokine storm or severe adverse events. Furthermore, it may be advantageous over treatment with type III IFN, given the systemic spread of SARS-CoV-2 from the lungs to the vasculature and other organs, as type I IFN receptors are expressed on virtually all cells, whereas type III IFN receptor expression is largely restricted to epithelial cells.

Michail Lionakis (NIAID, NIH) presented his recent work utilizing a kinase inhibitor to antagonize the COVID-19 cytokine storm ${ }^{16}$. The hyperinflammatory response in COVID-19 features activation of NF- $\kappa \mathrm{B}$ and the inflammasome (Nlrp3), as evidenced by the increased levels of proinflammatory cytokines and chemokines, including IL-1 $\beta$, TNF, IL-6, CCL2 and CCL3, among others. Consequently, he hypothesized that neutralizing these cytokines or their downstream signaling would be an effective strategy to suppress the COVID-19-associated cytokine storm.

Using mouse models of Bruton's tyrosine kinase (BTK) deficiency and pharmacological inhibitors of BTK, the Lionakis group previously pinpointed the target population of BTK inhibition in antifungal host defense to be macrophages, not $B$ cells. Ibrutinib is an effective and well-tolerated BTK inhibitor that blocks $\mathrm{B}$ cell receptor signaling and is used as a treatment for B cell malignancies and inflammatory conditions such as graft-versus-host disease. Thus, the suppression of macrophages has been considered a collateral effect of BTK inhibition, resulting in impaired fungal immunity as demonstrated by the susceptibility to fungal infections in a subset of ibrutinib-treated patients. Notably, ibrutinib has been reported to suppress macrophages by inhibiting the activation of both the inflammasome and NF- $\kappa \mathrm{B}$, precisely the two downstream pathways of the cytokine storm in severe COVID-19. These findings led Lionakis and his colleagues to hypothesize that BTK inhibitors would ameliorate hyperinflammation and thus prevent clinical deterioration in patients with severe COVID-19. The Lionakis group hypothesized that the overt immune response of SARS-CoV-2-infected alveolar macrophages could be inhibited at the proximal signaling level by the off-label use of a second generation BTK inhibitor, acalabrutinib. Such an approach could be superior to employing neutralizing antibodies specific to individual proinflammatory cytokines because it could potentially suppress the effect of multiple cytokines. In addition, because BTK is not expressed in T cells, BTK inhibition would limit the effect to macrophages without impacting anti-viral $\mathrm{T}$ cell effector function.

The results from the first clinical study with 19 patients were in line with the hypothesis, and some remarkable effects were observed for patients with COVID19 in the pre-ICU setting ${ }^{16}$. While patients with severe COVID-19 symptoms had increased production of IL- 6 by CD $14^{+}$ monocytes in blood, acalabrutinib treatment substantially improved oxygenation, decreased inflammation (for example, reduced production of CRP and IL-6) and also significantly increased lymphocyte numbers in the majority of patients. Mechanistically, it was found that monocytes but not B cells in patients with COVID-19 contained higher basal levels of phosphorylated BTK, validating that monocyte/macrophage-directed BTK inhibition provides the basis of suppressing the cytokine storm ${ }^{14}$. Currently, three different BTK inhibitors, including acalabrutinib, zanubrutinib and ibrutinib, are in clinical trials for treatment of severe COVID-19. Further studies will be required to validate these clinical findings, which, if confirmed, would potentially point to a broader use for BTK inhibitors in managing hyperinflammatory responses.

Collectively, the talks in this session highlighted the multifaceted approaches to neutralize cytokine storm in patients with COVID-19, and they showcased initial successes. Moreover, the results further illustrated the necessity of nimble 
approaches in applying interventional strategies, depending on the severity or stage of the diseases. Thus, the dosing, timing and the selection of the patient group must be considered carefully and in the context of disease progression. Dexamethasone treatment, for example, should be reserved for late-stage patients because of its immunosuppressive function, whereas type I IFNs are clearly suitable for investigation in immediate early treatment. Targeted suppression of immune cell activities by BTK inhibitors has been tested in patients requiring oxygen supplementation, but it remains unclear whether treatment at earlier stages would be even more effective.

\section{Concluding remarks}

This conference brought together over 1,600 scientists from different countries and different time zones, demonstrating the power of running a virtual conference but also documenting the immense worldwide interest in COVID-19 biology. Because of its great success and the enthusiastic feedback that was received, the next COVID-19 conference organized by the NIH/FDA Immunology Interest Group and Cytokine Interest Group is already being scheduled, and we look forward to broad participation in cyberspace in November 2020 to share the newest and most exciting research on COVID-19 with the international community.

Maja Buszko1, Jung-Hyun Park (D)2, Daniela Verthelyi (iD) ${ }^{3}$, Ranjan Sen ${ }^{4}$, Howard A. Young (D) ${ }^{5}$ and

Amy S. Rosenberg6凶

${ }^{1}$ Cellular Immunology Section, Laboratory of Immune System Biology, National Institute of Allergy and Infectious Diseases, National Institutes of Health, Bethesda, MD, USA. ${ }^{2}$ Experimental Immunology Branch, Center for Cancer Research, National Cancer Institute, National Institutes of Health, Bethesda, $M D$, USA. ${ }^{3}$ Laboratory of Innate Immunity, Division of Biotechnology Review and Research-III, Office of Biotechnology Products, Center for Drug Evaluation and Research, US Food and Drug Administration, Silver Spring, MD, USA. ${ }^{4}$ Laboratory of Molecular Biology and Immunology, National Institute on Aging, Baltimore, MD, USA. ${ }^{5}$ Laboratory of Cancer Immunometabolism, Center for Cancer Research, National Cancer Institute-Frederick, Frederick, MD, USA. ${ }^{6}$ Laboratory of Immunology, Division of Biotechnology Review and Research-III, Office of Biotechnology Products, Center for Drug Evaluation and Research, U.S. Food and Drug Administration, Silver Spring, MD, USA.

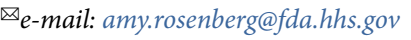

Published online: 27 August 2020

https://doi.org/10.1038/s41590-020-0779-1
References

7 1. Wrapp, D. et al. Science 367, 1260-1263 (2020).

2. Del Valle, D. M. et al. Nat. Med. https://doi.org/10.1038/s41591020-1051-9 (2020)

3. Diao, B. et al. Front. Immunol. 11, 827 (2020).

4. van de Veerdonk, F. L. et al. Elife https://doi.org/10.7554/ eLife.57555 (2020).

5. Ni, L. et al. Immunity 52, 971-977.e3 (2020).

6. Long, Q.-X. et al. Nat. Med. https://doi.org/10.1038/s41591-0200965-6 (2020).

7. Sekine, T. et al. Cell https://doi.org/10.1016/j.cell.2020.08.017 (2020).

8. Wu, L.-P. et al. Emerg. Infect. Dis. 13, 1562-1564 (2007).

9. Moratto, D. et al. J. Clin. Immunol. https://doi.org/10.1007/ s10875-020-00806-6 (2020).

10. Ginhoux, F. \& Guilliams, M. Immunity 44, 439-449 (2016).

11. Liao, M. et al. Nat. Med. 26, 842-844 (2020).

12. Bost, P. et al. Cell 181, 1475-1488.e12 (2020).

13. Jordan, M. B., Hildeman, D., Kappler, J. \& Marrack, P. Blood 104, 735-743 (2004)

14. Recovery Collaborative Group et al. N. Engl. J. Med. https://doi. org/10.1056/NEJMoa2021436 (2020).

15. Zhou, Q. et al. Front. Immunol. 11, 1061 (2020).

16. Roschewski, M. et al. Sci. Immunol. https://doi.org/10.1126 sciimmunol.abd0110 (2020).

17. Chatham, W. W. \& Cron, R. Q. J. Rheumatol. https://doi. org/10.3899/jrheum.200679 (2020).

Acknowledgements

We thank the speakers for their useful feedback in preparing this Meeting Report. The views expressed in this article are those of the authors and do not necessarily reflect the official policy or position of the United States Food and Drug Administration and the Department of Health and Human Services, nor does mention of trade names, commercial products, or organizations imply endorsement by the United States Government.

Competing interests

The authors declare no competing interests. 